\title{
Marcas de género en el diseño tipográfico de revistas de moda
}

\section{Flesler, Griselda}

Resumen:

Este trabajo analiza la hegemonía discursiva del campo tipográfico desde una perspectiva de género, y su incidencia en el diseño de revistas de moda. Se parte del supuesto que afirma que las prácticas del diseño tipográfico son espacios de construcción, reproducción y articulación de sentidos sobre las relaciones de género. De modo general, surge el interrogante sobre cómo se configuran las lecturas preferentes de una tipografía, qué hace que se la caracterice como femenina, masculina y/o neutra. Desde este enfoque, se retoman las críticas feministas a la producción discursiva del diseño moderno,

Cuadernos del Centro de Estudios de Diseño y Comunicación №76

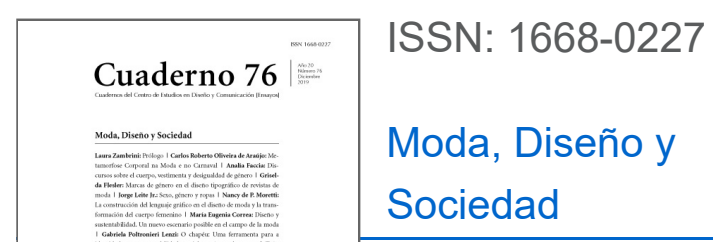

Año XX, Diciembre 2019, Buenos Aires, Argentina | 242 páginas

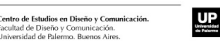

descargar PDF ver índice de la publicación

Ver todos los libros de la publicación

compartir en Facebook

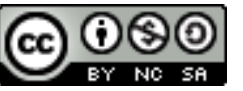

Esta obra está bajo una Licencia Creative Commons Atribución-NoComercialCompartirlgual 4.0 Internacional centrado en la funcionalidad y la neutralidad, con el objetivo de visibilizar las marcas de género existentes en el diseño, selección y clasificación de familias tipográficas para revistas de moda.

Palabras clave: diseño tipográfico - estudios de género - revistas de moda - revistas femeninas.

$\left(^{*}\right)$ La autora es Diseñadora Gráfica y Especialista en Diseño Comunicacional (FADUUBA). Profesora titular de la materia Diseño y Estudios de Género en FADU-UBA. Investigadora Principal del Instituto de Arte Americano (FADU-UBA) y Maestranda en Diseño Comunicacional en FADU-UBA.

Introducción

Este trabajo se inscribe en la intersección del diseño tipográfico para revistas de moda y los estudios de género. Dicha articulación, resulta un enfoque poco explorado para reflexionar sobre la construcción de estereotipos en las revistas denominadas "femeninas". Asimismo, se parte del supuesto que asume que esas publicaciones no sólo difunden lo que acontece en el campo de la moda y el diseño, sino que participan en su configuración. Se 
abordará particularmente el diseño tipográfico en las revistas de moda, para analizar cómo producen, seleccionan y categorizan lo femenino1 y los modos legítimos de hacerlo visible. Se intenta comprender a la tipografía inscripta en prácticas hetero-normativas, es decir, basada en un sistema binario, donde las únicas identidades posibles son la masculina y la femenina, siendo la primera más valorada que la segunda.

En esta instancia, surgen los siguientes interrogantes: ¿cuál es el canon de reglas que produce una fijación de algunas tipografías con estereotipos de género? ¿cuáles son las tipografías utilizadas para diseñar "lo femenino"? y finalmente ¿cómo la tipografía reproduce una tensión entre permisos y restricciones de las intervenciones feministas en el ámbito de la cultura masiva? Es decir, este artículo explora la hetero-tipografía (Flesler, 2015), o sea, las maneras en que la tipografía construye -y está construida por- la hetero-normatividad. En ese sentido, se considera que es la intersección de la perspectiva de género con la moda un telón de fondo propicio desde el cual abordar estos interrogantes. Dada la compleja e histórica relación entre el vestir y los estudios de género (Entwistle, 2002; Zambrini, 2010), un análisis de las revistas de moda femeninas desde el punto de vista del diseño tipográfico, intenta completar un área vacante. Marc Angenot (2010) señala en su análisis del discurso social, que existe "un canon de reglas", de lo decible y lo escribible, que determinan lo discursivamente aceptable en una época. En la hegemonía discursiva una sociedad se objetiva en textos y escritos, y en este trabajo se plantea que lo mismo sucede con los diseños. Es decir, se construye la pertinencia del uso de determinadas tipografías para diseñar lo "femenino". Aquí se considera que el discurso "heterotipográfico" se inicia con el surgimiento del Movimiento Moderno2 a principios del siglo XX, y se consolida en el período de entreguerras, momento clave del afianzamiento y profesionalización del campo disciplinar, así como también de las revistas de moda femeninas, a partir del éxodo europeo a los Estados Unidos.

Los estudios de género son una categoría útil para el análisis tipográfico, en tanto nos permiten desnaturalizar los valores y marcas genéricas asociadas a diferentes estilos. Asimismo, proveen herramientas teóricas para la deconstrucción de los estereotipos respecto a la funcionalidad, la legibilidad y la pertinencia del uso de determinadas tipografías, según el contexto. Los debates actuales en cuanto al género cómo categoría de análisis, en general se desarrollaron a partir del trabajo de Joan W. Scott (1999, [1986]) quien sostiene que el género consiste en buscar las cualidades sociales de las distinciones basadas en el sexo. Para Scott, el género es el elemento fundamental de las relaciones significantes de poder, asentado en las diferencias que se perciben entre los sexos y, a partir del cual se establecen las redes de poder. Los análisis posteriores que repiensan la noción de género provienen, entre otras autoras, de Judith Butler, quien desestabiliza conceptos como mujer y varón para mostrar de qué manera la realidad socio-cultural los sujeta discursivamente, produciendo sus cuerpos en y dentro de las categorías de sexo binario, originario y naturalizado. Para esta autora, el modelo discursivo/epistémico hegemónico de inteligibilidad de género es la heterosexualidad: "una rejilla de inteligibilidad cultural a través de la cual se naturalizan cuerpos, géneros y deseos” (2007, [1990], p. 292)3 .

Desde la década de 1980, diversas perspectivas de los estudios de género han sido un modo útil de interpelación de la hegemonía discursiva del campo del diseño gráfico. Aquí se parte del supuesto de que el campo tipográfico es atravesado por el modelo discursivo hegemónico de la heterosexualidad -por ello el término "hetero-tipografía"-, y como consecuencia existe una jerarquización y valorización de determinados estilos y morfologías por sobre otras.

Para el análisis de las cuestiones mencionadas anteriormente, este artículo se organiza en dos partes. La primera recupera el "canon de reglas" de la hegemonía discursiva del campo tipográfico, establecido por el 
Movimiento Moderno, en un contexto racional y funcionalista, centrado en la producción, la funcionalidad y la síntesis de la forma. Asimismo, se argumenta que existen tipografías que representan "la buena forma", es decir, geométrica, sintética, racional y funcional, generalmente asociadas al estereotipo de lo masculino, en oposición a "la otredad" tipográfica inscripta en morfologías "decorativas", en línea con lo femenino y lo afeminado y, por consiguiente, con un menor grado de legitimidad. Esta perspectiva retoma la crítica feminista a la hegemonía discursiva del Movimiento Moderno de autoras como Judy Attfield (1989) y Sheila Levrant de Bretteville (1973). Específicamente, en el campo tipográfico, se tiene en cuenta lo expuesto por Kattie Salen (2001) respecto a la construcción ficticia de un lenguaje universal y lo trabajado por Leslie Savan (2001 [1976]), para establecer el origen del vínculo entre el lenguaje funcionalista y la construcción de un discurso comunicacional con pretensión de universalidad.

En la segunda parte del trabajo, se analiza concretamente cómo el "canon de reglas" se articula en el diseño tipográfico de las revistas de moda a partir de la reproducción de estereotipos de género. En esta línea, se retoma, por un lado, el aporte de Morna Laing (2014) en su análisis de la revista Lula girl. Aunque la autora no trabaja específicamente la tipografía, su aporte conjuga el análisis cultural, la teoría de género y la sociología de la moda. Laing, hace foco en dos conceptos claves de Barthes (1967) para pensar las representaciones de lo femenino en el campo de la moda: lo sublime y lo inocente (Laing, 2014, p. 272). Un marco pertinente para analizar qué elecciones tipográficas toman los diseñadores de las revistas de moda para responder a estas facetas. Por otro lado, se analizan algunos casos de revistas de moda en los que la elección tipográfica responde a un intento por despegarse de los estereotipos de la feminidad tradicional (pasividad, ámbito privado, sensibilidad, maternidad, elegancia, etc.). Para ello, se analizará la configuración de la "mujer actual" y sus derechos adquiridos en algunos ejemplos de revistas femeninas contemporáneas. Es decir, el modo en que el "feminismo femenino" (Groeneveld, 2009) se traduce en la elección de determinadas tipografías deudoras del Movimiento Moderno.

Hetero-tipografía y la hegemonía discursiva

Se puede situar el origen del diseño tipográfico contemporáneo a principios del siglo XX, en el denominado período de entreguerras en Europa, momento en el que se escinde definitivamente del oficio de la impresión y la fundición de tipos. En aquel contexto, el Movimiento Moderno, de gran influencia en escuelas como la alemana Bauhaus, conceptualizó la "buena forma" como una morfología funcionalista ligada a la producción industrial y al positivismo (Devalle, 2009). Las vanguardias abstraccionistas y constructivistas fueron una gran influencia en la definición de un canon morfológico geométrico, sintético y carente de rasgos individuales, gestuales o decorativos. El diseño tipográfico también adoptó estas características. Luego de algunos intentos elaborados en la Bauhaus por Josef Albers y Herbert Bayer, se promovió el uso de las fuentes denominadas "de palo seco" o "san serif"4 como las más adecuadas para la composición, no solamente de titulares, sino también de texto corrido. En el año 1925, Jan Tschichold definió la forma legítima del diseño tipográfico: funcionalidad y síntesis, todo formalismo intuitivo debería ser evitado. Este movimiento, denominado "la Nueva Tipografía" estableció la asociación entre las morfologías simples con una retórica de lo universal y, por lo tanto, de la neutralidad (Tschichold, 2001, [1930]). Asimismo, sentó las bases para futuros proyectos de carácter neutralistas tales como los exitosos alfabetos diseñados a partir de la segunda posguerra. Este es el caso de la familia Univers, diseñada por el suizo Adrian Frutiger en el año 1957, la cual desde su nombre ya instalaba una utopía universalista o, bien, la Hélvética, diseñada en el mismo año por Max Miedinger, otro afamado tipógrafo suizo "con la intención de crear un carácter tipográfico perfectamente neutral sin formas abiertamente individuales ni 
idiosincrasias personales" (Savan, 2001 [1976], p. 309). En este sentido, resulta útil el concepto llamado falso neutro (Bessa, 2008) para mostrar cómo en el campo tipográfico lo neutral aparece con morfologías generalmente asociadas a lo masculino. Esta falsa neutralidad, propia de la hegemonía discursiva (Angenot, 2010) del Movimiento Moderno, ha establecido una jerarquía de legitimidades, es decir, un "canon de reglas" en el campo tipográfico. Específicamente, autoras como Judy Attfield (1989), Cheryl Buckley (1989) y Pat Kirkham (2000; 1996; 1989) echaron luz sobre las consecuencias políticas de pensar la producción de los objetos como universales o neutrales, despojados de toda relación histórica y social. La crítica feminista hizo evidente que el canon es pensado como un valor universal y neutro, a la vez que deja por fuera otros discursos, prácticas y sujetos en detrimento de lo femenino (Zambrini y Flesler, 2017). Según Judy Attfield (1989), la concepción funcionalista dominante priorizó a la máquina sobre el cuerpo, es decir: la forma (femenina) debía seguir a la función (masculina)5. Desde una crítica más reciente, Kattie Salen argumenta que fue el Movimiento Moderno quien estableció que una buena tipografía era aquella "neutral" y "transparente", es decir, carente de distinciones particulares, decorativas, étnicas, etc. La autora sostiene que este es un legado clasista y sexista, en la medida que la forma neutra no existe como tal, sino que simplemente responde a la forma hegemónica establecida por diseñadores europeos en la década del 20 y 30 del siglo XX. Con todo lo expuesto, aquí se destaca la existencia de un linaje de familias tipográficas asociadas al concepto de universalidad, pureza y neutralidad cuyo resultado debía ser garantía de máxima legibilidad. Como se ha mencionado en trabajos anteriores (Flesler, 2015; 2016), estas tipografías validadas y jerarquizadas responden morfológicamente al mapa de significaciones asociadas a un imaginario masculino (funcional, racional, frío, neutro, limpio, recto, serio) e ilustran acerca de la concepción de la neutralidad en el diseño. Es decir, dicha retórica neutral ha sido significativa en el campo del diseño y, en consecuencia, lo ha sido también en su modo ideológico de concebir los géneros.

Diseño tipográfico en las revistas de moda: tradiciones y tensiones

En la década de 1930 y 1940, la inmigración europea fue significativa en el campo del dise- ño estadounidense, en el contexto de consolidación de la disciplina. No sólo, se incorporaron individualidades sino también escuelas o tendencias enteras (Satué, 1993, p. 258). En el caso de las revistas de moda, la dirección artística fue asumida por diseñadoras y diseñadores, cuya sólida formación significó un salto cualitativo en el diseño editorial de revistas como Harper's Bazaar, Vogue yVanity Fair, particularmente en la fotografía y la tipografía.

En el caso de Vogue y Vanity Fair, por ejemplo, fue fundamental la llegada de Mehemed Fehmy Agha de origen turco-ruso, en 1929. Siendo pintor, fotógrafo y tipógrafo, profesionalizó el diseño editorial en la medida que logró que fuera parte del proceso de producción de la publicación y no un agregado posterior al diseño de contenidos. Esto es fundamental para entender el modo en que la elección tipográfica comenzó a considerarse una pieza clave de la construcción gráfica/discursiva de una publicación.

En este contexto, puede observarse un intento de sistematización del programa tipográfico de algunas publicaciones, aunque todavía eran muchas las revistas de moda cuya elección tipográfica respondía a un ilustrador o un número particular (y no a un sistema de diseño cuyas constantes y variables estén pautadas). En ese período, la totalidad de los logos de las revistas de moda pasaron de letras ilustradas a tipografía sestilo art deco, serif y san serif, número a número sin un criterio editorial. Es interesante observar como en su mayoría, las tipografías utilizadas respondieron a la lógica que equiparó "lo femenino" con lo decorativo y con aquellas tipografías de gesto caligráfico, manual como serif (Ver Figura 1). En el marco de una fuerte impronta de las tipografías san serif como signo de modernidad, éstas fueron destinadas a otros programas de diseño siendo 
poco utilizadas en las revistas de moda de la época. Es entonces, que en el período de profesionalización del diseño editorial de revistas de moda, se reproduce a través de las tipografías, un fuerte estereotipo tipográfico que perdura hasta la actualidad.

Fue en la década de 1940, cuando el diseño del nombre de la mayoría de las publicaciones se estabilizó y se definió con una sola tipografía. La idea del nombre de la publicación como marca sólida surgió en paralelo con un incipiente mercado publicitario de la segunda posguerra. Asimismo, fue el nacimiento de una tradición tipográfica de las revistas de moda que respondía fielmente a la necesidad de una "tipografía femenina, elegante, con estilo". Esta tradición se basó en la recuperación de las tipografías denominadas didonas.

La invención de la tradición tipográfica de moda

Abbott Miller (2007) analiza cómo los patrones culturales son capaces de definir el modo en que entendemos las tipografías y explica cómo este fenómeno es observable en el campo de la moda, donde al igual que lo observado en las distribuidoras, las revistas de moda han articulado un panorama de estereotipos sexogenéricos en torno a las tipografías serif y san serif. Uno de ellos fue la asociación tácita entre las tipografías didonas con lo femenino. Se denomina tipografías didonas debido a la combinación entre Didot y Bodoni, las familias más paradigmáticas de su especie6 . Las tipografías de finales del siglo XVIII y principios del siglo XIX del francés Firmin Didot y el italiano Giambattista Bodoni, se caracterizaban por el altísimo contraste entre sus astas finas y gruesas, así como por sus finos remates. Esta característica se pudo lograr básicamente gracias a los avances tecnológicos que permitieron la impresión de trazos muy finos. Después de estar en desuso durante el siglo XIX, las tipografías Bodoni y Didot hicieron un regreso a principios del siglo XX, en parte porque su morfología geométrica parecía moderna nuevamente. En el año 1912, la fundidora Deberny + Peignot, compró los punzones originales de Didot, haciendo la tipografía nuevamente accesible a los diseñadores. Esto permitió que, en los años de la primera posguerra, su uso fuera muy frecuente por aquellos diseñadores conocedores de su tradición. Esto se dio principalmente en Estados Unidos, destino de muchos artistas, diseñadores e intelectuales provenientes de Europa. Allí se los recibió con un significativo interés por un mercado editorial que necesitaba sistematización y profesionalización. Fue en este contexto que se inscribió el trabajo de directoras y directores de arte de revistas de moda que adoptaron el lenguaje de las tipografías didonas como sinónimo de elegancia, femineidad y moda. Algunos ejemplos son revistas como Glamour, Seventeen, Charmy Mademoiselle bajo la dirección de arte de Cipe Pineles7 . Otros ejemplos paradigmáticos son las revistas Vogue, dirigida por Mehemed Fehm y Agha de 1929 a 1942 y Harper's Bazaar diseñada por Alexey Brodovitch desde 1934 hasta 1958. (Ver Figura 2)

Según Miller (2007), la conexión entre Didot, Bodoni y la industria de la moda está tan arraigada que, no sólo, Vogue y Harper's Bazaar continúan utilizándolas hasta el presente, sino también otras innumerables revistas y marcas de productos ligados al campo de la moda. Un claro ejemplo de ello es el reciente paquete de tipografías estilo didonas y denominado Revista, diseñado en 2015 para la distribuidora Latinotype por Paula Nazal Selaive, Marcelo Quiroz y Daniel Hernández. (Ver Figura 3)

Tensiones en torno a "lo femenino" en la tipografía: de la "femineidad tradicional" a la "mujer actual"

El diseño tipográfico en las revistas de moda es un terreno propicio para analizar las tensiones y cambios sobre "lo femenino", entendiendo al mismo como un constructo cultural, históricamente determinado. Si se considera 
las políticas feministas de las últimas décadas, es pertinente observar la influencia que ha tenido la denominada "tercera ola" del feminismo en las revistas de moda y su diseño gráfico. Elizabeth Groeneveld sostiene que a partir de 1980 tendencias tales como el "fashionable feminism" y el "girlie feminism" han ganado terreno en el mundo de las revistas femeninas (Ver Figura 4). Este fenómeno permite analizar las posibilidades de inserción de los feminismos en este tipo de publicaciones, a la vez que pone en tensión las posibles simplificaciones de dichos movimientos con la oportunidad de masificar demandas históricas en la cultura popular (Groeneveld, 2009). Desde el punto de vista del diseño tipográfico, aquí se considera pertinente focalizar que en el camino por diferenciarse de las revistas femeninas tradicionales, se han dejado de utilizar las tipografías didonas, entendiendo a éstas como representantes de un mercado de la moda al que se quiere cuestionar. Aparecen entonces en el logo de estas revistas, las tipografías san serif, que como se ha señalado anteriormente, remiten al universo funcional y productivo asociado históricamente a la masculinidad y que hoy remite "a la mujer real". En la configuración contemporánea de "lo femenino", además del perfil productivo de la mujer actual, subyacen otras lecturas posibles. Simone de Beauvoir (1949) afirmaba que las mujeres están marcadas como "eternas niñas" en términos de su pasividad, inferioridad y dependencia con el varón7 . En su trabajo reciente, Morna Laing asegura que desde la década de 1980, la figura de la "niña" se ha convertido en una de las más importantes posiciones de las revistas de moda. Y afirma, siguiendo a Roland Barthes (1967) que esta forma de construir la feminidad mítica propia de occidente, a partir de lo sublime y lo infantil, se reproduce en las revistas de moda contemporáneas (Laing, 2014; 2012). Laing analiza la revista Lula Girl como máximo exponente de este fenómeno. Su análisis se centra en las imágenes de la publicación y su línea editorial. Acorde al objetivo del presente trabajo, se puede observar cómo las tipografías utilizadas en Lula Girl (Ver Figura 5) reproducen los estereotipos de inocencia y femineidad que se destacan anteriormente en los resultados por búsqueda de términos como "femenino" o girlie" en las distribuidoras de tipografías contemporáneas, antes mencionadas.

Pareciera entonces que "lo femenino" en las revistas de moda contemporáneas, se tensiona entre una mujer "feminista femenina" y una mujer "infantil e inocente". Un ejemplo local en el que se puede observar esta doble dimensión es la revista Ohlalá! (Ver Figura 6). Por un lado, su logo en tipografía san serif respondería a una mujer contemporánea, productiva, en oposición a la mujer tradicional. Por otro lado, se observa un lenguaje espontáneo de flechitas y marcaciones que simulan lo manual, así como el uso de la "tipografía gestual" presente en la bajada del logo con la inscripción "comunidad de mujeres"- que constituye un universo aniñado e infantil propio de los dibujos de una agenda preadolescente.

\section{A modo de cierre}

Hasta aquí este trabajo intentó presentar algunos ejes de análisis que posibilitan el encuentro entre la historia de la tipografía, las revistas de moda y la crítica feminista al campo del diseño. Dicho encuentro ha permitido poner en cuestión algunas categorías binarias que han sustentado la modernidad y sus legados en el campo del diseño tipográfico. En ese recorrido, sobresalió la necesidad de repensar el impacto del legado y la vigencia de los principales conceptos del Movimiento Moderno. En este sentido, recobra vitalidad discutir sobre los modos de clasificación tipográfica y la reproducción de estereotipos de género en las publicaciones femeninas. Se intentó echar luz sobre cómo el desarrollo del campo de la tipografía estuvo atravesado por ideologías de género que determinaron, la mayor o menor valoración, de distintos estilos de diseño por encima de otros. En consecuencia, se analizó la hegemonía discursiva del campo tipográfico, entendida como hetero-tipografía, es decir, las maneras en que la tipografía construye y a la vez, está atravesada por la heteronormatividad y el pensamiento binario. 
En el análisis específico del diseño tipográfico de revistas de moda, se describió cómo se estableció un estereotipo de "lo femenino" reproducido en las tipografías denominadas "didonas", en el contexto de la profesionalización del diseño editorial del período entreguerras en Estados Unidos, cuyo legado continúa hasta el presente.

Asimismo, la dimensión de "lo femenino" en las últimas décadas puede verse impactado en el diseño gráfico de las revistas de moda por la denominada "tercera ola de revistas" (Groeneveld, 2009), reproducida en tipografías san serif, así como también, por una asociación con la inocencia y femineidad y las morfologías despreciadas por el Movimiento Moderno. Finalmente, de lo que se trata es de poner en evidencia la naturalización con la que generalmente son incorporados los modos de validación y clasificación al momento de diseñar un producto editorial. El propósito de este trabajo, lejos de clausurar el tema, ha sido dejar planteadas cuestiones relevantes sobre el campo tipográfico desde una mirada de género, a fin de visibilizar el carácter ideológico propio de la hegemonía discursiva de la disciplina.

Notas

1. En este trabajo se emplea el término "femenino" para referirse al conjunto de características definidas culturalmente. Para una distinción entre los términos feminista, femenino y femenil, ver: Moi, T. (1989).

2. Este movimiento se inscribe en el funcionalismo, "término utilizado por teóricos del diseño para describir las ideas de los protagonistas del movimiento moderno de principios del siglo XX en arquitectura, que trataban de obtener la forma a través de una consideración abstracta de la función (...) en lugar de añadir ornamentos a la superficie para hacerlo más deseable" (Sparke, 2015 [1986], p. 246).

3. Acerca del enfoque post-estructuralista sobre las nociones de sujeto e identidad femenina véase: Zambrini e ladevito (2009).

4. En tipografía, un tipo de letra de palo seco, sans serif o sin remates es aquel en el que cada carácter carece de las pequeñas terminaciones llamadas remates, gracias o serifas.

5. Attfield de-construye desde una perspectiva feminista la famosa frase de Sullivan (1896) "La forma sigue a la función".

6. Este neologismo fue creado en la década de 1950 por Maximillien Vox, autor de una de las clasificaciones tipográficas más reconocidas, adoptada en 1962 por la Association Typographique Internationale (ATypl).

7. Acerca de la biografía de Cipe Pineles puede consultarse: Scotford, 1999. Unos años mas tarde, se publica en Buenos Aires por primera vez una breve reseña de su trabajo en la revista Tipográfica, publicación especializada en tipografía y dirigida por Rubén Fontana (Flesler, 2002).

8. Citado por Morna Laing (2012, pp. 22-23)

Bibliografía 
Angenot, M. (2010). El discurso social. Los límites históricos de lo pensable y lo decible. Buenos Aires: Siglo $\mathrm{XXI}$.

Attfield, J. y Kirkham, P. (1989). A view from the interior: Feminism, Women and Design. Londres: The Women's Press.

Attfield, J. (1989). Form/female follows function/male: feminist critiques of design. En: Walker, J. (comp.) Design History and the History of Design.Londres: Pluto Press.

Barthes, R. (1990). Sistema de la moda. Barcelona: Gustavo Gili.

Bessa, P. (2008. Skittish skirts and scanty Silhouettes: The tribulations of Gender in Modern Signage. En: Visible Language 42.2. Pp. 119-141. Rhode Island: Rhode Island School of Design.

Buckley, Ch. (1989). Made in Patriarchy: Toward a Feminist Analysis of Women and Design. En: Margolin, V. (comp.) Design discourse: history, theory, criticism. Pp. 251-262. Chicago: The University of Chicago Press.

Butler, J. (2007 [1990]). El género en disputa. El feminismo y la subversión de la identidad. Barcelona: Paidós.

de Beauvoir, S. (2000 [1949]). El segundo sexo. Madrid: Cátedra.

Devalle, V. (2009). La travesía de la forma. Emergencia y consolidación del diseño gráfico (1948-1984). Buenos Aires: Paidos.

Entwistle, J. (2002). El cuerpo y la moda. Una visión sociológica. Paidós, Barcelona.

Flesler, G. (2016). Tipografía y estudios de género: estereotipos, modelos de clasificación y nuevas tecnologías. En: Actas de XXX Jornadas de Investigación: Configuraciones, acciones y relatos. Buenos Aires. FADU-UBA. . (2015). Diseño y nuevas tecnologías: Una mirada desde los estudios de género al diseño, selección y categorización de tipografías en las plataformas digitales. En: Actas $3^{\circ}$ Congreso Virtual: las Nuevas Tecnologías. Su influencia en la formación y producción disciplinar. San Miguel de Tucumán: Facultad de ArtesUNT. . (2002) Cipe Pineles: Femenino y singular. En: Revista tipográfica nº 54. Buenos Aires.

Groeneveld, E. (2009). Be a feminist or just dress like one: Bust, fashion and feminism as lifestyle. En: Journal of genderStudies. Vol. 18. N 2. Pp. 179-190.

Kirkham, P. (ed.) (2000). Women Designers in the USA, 1900-2000: Diversity and Difference. Nueva York:Yale University Press.

(ed.) (1996).The gendered Object. Manchester y Nueva York: Manchester University Press.

Laing, M. (2014). The Lula girl as sublime and childlike: nostalgic investments in contemporary fashion magazines. En: Critical Studies in Fashion and Beauty. 5:2. Pp. 271-293. 

(2012). Heavenlycreatures in Vogue: childlikefemininity and longingforinnocencelost. En: Lifter, R. (ed.) WorkingPapers in FashionStudies. Pp 17-32. Londres: London College of Fashion.

Levrant de Bretteville, S. (2001 [973]). Algunos aspectos del diseño desde la perspectiva de una diseñadora. En: Bierut, M. y otros (comp.) Fundamentos del Diseño Gráfico. Buenos Aires: Ediciones Infinito.

Moi, T. (1989). Feminist, Female, Feminine. En: Belsey, C. y Moore, J. (eds.) The Feminist Reader: Essays in Gender and the Politics of Literary Criticism. Pp. 117-132. Londres: Macmillan.

Miller, A. (2007). Throughthick and thin: fashion and type. En: Eye no. 65 vol. 17.

Salen, K. (2001). Surrogate Multiplicities: Typography in the Age of Invisibility. En: Visible Language, Vol. 35, No. 2. Pp. 132-153.

Savan, L. (2001) [1976]. Este carácter tipográfico está cambiando tu vida. En: Bierut, M.(comp.) Fundamentos del Diseño Gráfico, Pp. 308-312. Buenos Aires: Ediciones Infinito.

Scotford, M. (1999). CipePineles: A life of Design. Nueva York: W. W. Norton Company.

Scott, J. W. (1999 [1986]). El género: una categoría útil para el análisis histórico. En: Navarro, M. y Stimpson, C. (comps.) Sexualidad, género y roles sexuales. Pp. 37-75. Buenos Aires: FCE.

Sparke, P. (2015 [1986]). Diseño y cultura una introducción. Desde 1900 hasta la actualidad. Barcelona: G. Gili.

Sullivan, L. H. (1896). The Tall Office Building Artistically Considered. En: Lippincott's Magazine (pp. 403-409).

Filadelfia. Recuperado el 20/01/2017 de: https://ocw.mit.edu/ courses/architecture/4-205-analysis-ofcontemporary-architecture-fall-2009/readings/MIT4_205F09_Sullivan.pdf

Tschichold, J. (2001 [1930]). Nueva vida impresa. En: Bierut, M.(comp.) Fundamentos del Diseño Gráfico, Buenos Aires: Ediciones Infinito.

Zambrini, L. e ladevito, P. (2009). Feminismo filosófico y pensamiento post-estructuralista: teorías y reflexiones acerca de las nociones de sujeto e identidad femenina" en Sexualidad, Salud y Sociedad. En: Revista Latinoamericana, N. 2. Pp.162-180. Brasil.

Zambrini, L. (2010). "Modos de vestir e identidades de género: reflexiones sobre las marcas culturales sobre el cuerpo". Revista de Estudios de Género Nomadías, 11. Santiago de Chile: Universidad Nacional de Chile. Disponible en: http://www.nomadias.uchile.cl/ index.php/NO/article/viewFile/15158/15574

Zambrini, L. y Flesler, G. (2017). Perspectiva de Género y diseño: Deconstruir la neutralidad de la tipografía y la indumentaria. Revista Inclusiones. Vol. 4. Num. Especial, Julio- Septiembre, ISSN 0719-4706, pp. 11-22. Santiago de Chile.

Abstract: This paper analyzes the discursive hegemony of the typographic field from a gender perspective, and its incidence in the design of fashion magazines. It is based on the assumption that typographic design practices 
are spaces for construction, reproduction and articulation of meanings about gender relations. In general, the question arises about how the preferred readings of a typeface are configured, what makes it characterize as feminine, masculine and / or neutral. From this perspective, feminist critiques of modern design discursive, focusing on functionality and neutrality, are use in order to make visible the existing gender marcs in the design, selection and classification of typographic families for fashion magazines.

Key words: typographic design - gender studies - fashion magazines - women's magazines.

Resumo: Este trabalho analiza a hegemonía discursiva do campo tipográfico desde uma perspectiva de género e seu impacto no projeto de revistas de moda. Parte-se do suposto que afirma que as práticas do design tipográfico são espaços de construção, reprodução e articulação de sentidos sobre as relações de género. De modo geral, surge a interrogante sobre como configuram-se as leituras preferenciais de uma tipografía, o que faz com que esta seja caracterizada como femenina, masculina e/ou neutra. Desde este enfoque, retomam-se as críticas feministas à produção discursiva do design moderno, centralizado na função e a neutralidade, com o objetivo de visibilizar as marcas de género existentes no design, seleção e classificação de familias tipográficas para revistas de moda.

Palabras chave: design tipográfico - estudos de género - revistas de moda - revistas femininas

[Las traducciones de los abstracts fueron supervisadas por el autor de cada artículo]

Marcas de género en el diseño tipográfico de revistas de moda fue publicado de la página 49 a página61 en Cuadernos del Centro de Estudios de Diseño y Comunicación №76 